\title{
On the two ways for the computing of the fire front positions and the rate of spread
}

\author{
K. Chetehouna ${ }^{1}$, I. Zarguili ${ }^{1}$, O. Séro-Guillaume ${ }^{2}$, \\ F. Giroud ${ }^{3} \&$ C. Picard ${ }^{3}$ \\ ${ }^{1}$ Institut PRISME UPRES EA 4229 EP-RES, \\ Université d'Orléans/ENSI de Bourges, 88 bd Lahitolle, \\ 18020 Bourges cedex, France \\ ${ }^{2}$ LEMTA (UMR 7563 CNRS/INPL/UHP) 2, avenue de la Forêt de Haye, \\ 54504 Vandoeuvre les Nancy cedex, France \\ ${ }^{3}$ Centre d'Essai et de Recherche de l'Entente, Sécurité Civile Valabre, \\ 13120 Gardannes, France
}

\begin{abstract}
The aim of this paper is to propose two methods for the identification of the fire front positions and the rate of spread in order to validate the first one by the second method. The first technique is based on the heat fluxes measured by specific thermal sensors and the use of the inverse method. The second one uses an image processing technique to obtain the fire front positions in pixels. The real positions in the propagation zone are determined using a matrix of passage calculated by applying the simple direct linear transformation (DLT) algorithm. The two methods give promising results and show that the thermal sensor technique can be validated by the image processing method.

Keywords: heat flux sensor, flame model, image processing, fire front positions, rate of spread, flame characteristics.
\end{abstract}

\section{Introduction}

Because of their geographical location and their climatic conditions, countries such as Spain, Portugal, Italy, France and Greece are the Member States of the European Union most exposed to forest fires. 
During the year 2005, in these five countries, 589559 hectares were flaring shared in $85 \%$ between Portugal and Spain. Recently in summer 2007, Greece knew the most fatal forest fire of Europe making 64 died and 200000 hectares of surface flaring. These countries invest more than 2.5 billion euros per year for the prevention and elimination of wildfires, although that did not make possible to decrease the extent of the surfaces traversed by fire. However, it is necessary to conduct fundamental and applied research to improve the understanding and ability to predict the behaviour of free-burning vegetation fires, which allows an increasingly effective forest fire management.

Several methods were already developed to study the behaviour and some characteristics of fire. It can be given by a development of a mathematical model [1-3] and can also be done from the analysis of the video images in experiments of fire. Actually, some research is leaned for using the technique of video image processing, this has shown some promising possibilities for determining the position or the rate of spread $(R O S)$ of fires [4-6]. Pastor et al. [4] have proposed a new image processing method based on Direct Linear Transformation (DLT) [7], using an infrared camera, for computing the positions and the ROS for a linear flame front propagated on a flat surface.

In this paper we propose a method based on measure of the radiative heat flux for determining the characteristics and positions of fire front and the rate of spread using the inverse method [8]. Also, the identified fire front positions are validated by the image processing technique based on the method developed in [4] using a visual video camera employed by the fire-fighters in real situation. The second section is devoted to the description of the experimental set-up and the heat fluxes sensor. We will present in this paper the specifications of the sensor and its transfer function. A detailed description of this apparatus was given in [8]. In third section we will present the method of identification of fire front positions and flame characteristics based on the heat fluxes measurement and on the inverse method. The fourth section is dedicated to the description of the image procession technique. The comparison of these two ways of identification was presented in the last section of this paper.

\section{Experimental set-up and heat fluxes sensor}

The experimental device (figure.1) was set up in a laboratory of the firemen departmental School of Sancoins in France. Experimental tests were conducted in a table of combustion manufactured in Siporex material. This table had $2 \mathrm{~m}^{2}$ useful burning surface $(2 \times 1 \mathrm{~m})$ and a depth of $0.2 \mathrm{~m}$. Indeed, the whole of the experimental device consists of fuel bed, video camera, wireless heat fluxes sensor and 2 plasticized threads placed each one in distance of $20 \mathrm{~cm}$ of the extremity of the table. The fuel bed used in this study is straw with a load of 1.2 $\mathrm{kg} / \mathrm{m}^{2}$.

The wireless thermal sensor in figure. 1 consists in a steel body, a thin heat insulating layer, a glue layer, 4 copper plates and 9 type $\mathrm{K}$ thermocouples. A previous theoretical study and experiments have been carried out to represent the thermal behaviour of each side of the sensor with a resistor-capacitor model. 


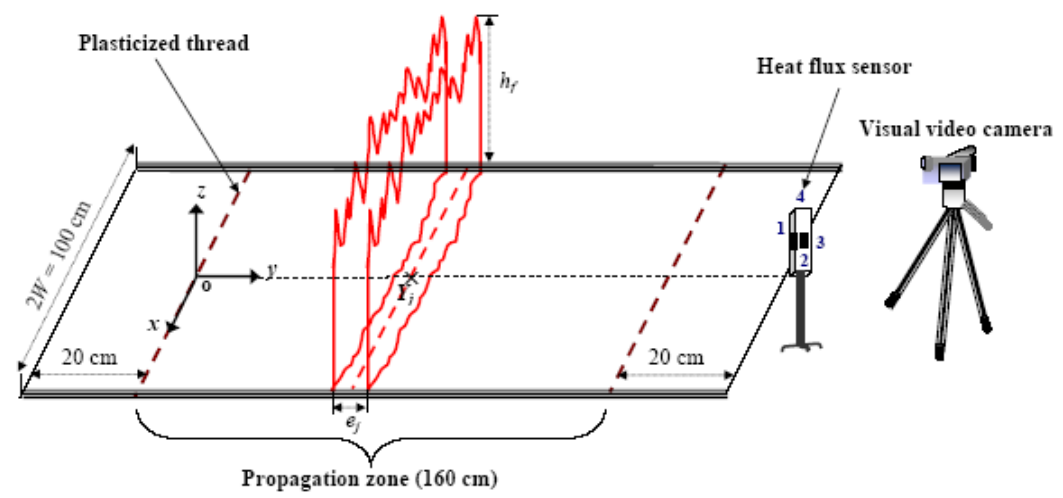

Figure 1: $\quad$ Experimental set-up.

$$
\varphi_{\mathrm{cu}}(t)=\left(C_{1} \frac{d}{d t}+\left(\frac{1}{R}+\frac{1}{R_{e_{1}}}\right)\right) \theta_{\mathrm{cu}}(t)-\frac{1}{R} \theta_{\mathrm{ac}}(t)
$$

The model (1) allows to calculate the absorbed heat flux by the copper plate, $\varphi_{\mathrm{cu}}$, from measured temperatures, $\theta_{\mathrm{cu}}$ (the copper plate temperature) and $\theta_{\mathrm{ac}}$ (the sensor body temperature). Parameters of the transfer function for sensor $\left(C_{1}, R\right.$, $R_{\mathrm{e} 1}$ ) have been obtained by calibration experiments [9]. The values of these parameters are given in table 1.

Table 1: Values of the parameters of the transfer function.

\begin{tabular}{|c|c|c|c|}
\hline$R_{\mathrm{e} 1}(\mathrm{~K} / \mathrm{W})$ & $R(\mathrm{~K} / \mathrm{W})$ & $C_{1}(\mathrm{~J} / \mathrm{K})$ & $R_{\mathrm{e} 2}(\mathrm{~K} / \mathrm{W})$ \\
\hline 23.7 & 21.7 & 10.4 & 20.9 \\
\hline
\end{tabular}

\section{Heat fluxes method of identification}

The thermal sensor is placed in the middle of the fire table on top of the vegetation (figure.1). The theoretical heat fluxes, emitted by the flame and received by the different faces of the sensor, were calculated through the models developed in a preceding study [8].

- Face 1 (front face):

$$
\Phi_{a}^{\text {th }}\left(Y_{j}, e_{j}, h_{f}, T_{f}, K_{f}\right)=2 K_{f} \frac{B}{\pi} \int_{Y_{j} \frac{e_{j}}{2}}^{Y_{j}-\frac{e_{j}}{2}} \int_{f}^{0} T_{f}^{4} \frac{Y}{X^{2}+Y^{2}} \sin \theta_{f} d X d Y
$$

- $\quad$ Face 2 and 4 (lateral faces):

$$
\Phi_{l}^{t h}\left(Y_{j}, e_{j}, h_{f}, T_{f}, K_{f}\right)=2 K_{f} \frac{B}{\pi} \int_{Y_{j}+\frac{e_{j}}{2}}^{Y_{j}} \int_{W}^{\frac{e_{j}}{2}} T_{f}^{4} \frac{X}{X^{2}+Y^{2}} \sin \theta_{f} d X d Y
$$

These equations consider that the flame is assimilated to a parallelepiped with a variable thickness $e_{j}$, characterized by a height $h_{f}$, a temperature $T_{f}$, an extinction 
coefficient $K_{f}$ and their positions $Y_{j}$. The rate of spread $R O S$ can be obtained by a least-squares regression based on the values of these positions.

The experimental heat fluxes measured in section 2 will be used with the formulas (2) and (3) to identify the different parameters described above. For these, an objective function $O b j$ will be minimized:

$$
\begin{aligned}
\operatorname{Obj}(\eta) & =\sum_{j=1}^{N}\left(\Phi_{a}^{t h}\left(\eta_{j}\right)-\Phi_{1}^{\exp }\left(t_{j}\right)\right)^{2}+\sum_{j=1}^{N} \alpha_{j}^{2}\left(\Phi_{l}^{t h}\left(\eta_{j}\right)-\Phi_{2}^{\exp }\left(t_{j}\right)\right)^{2} \\
& +\sum_{j=1}^{N} \beta_{j}^{2}\left(\Phi_{l}^{\text {th }}\left(\eta_{j}\right)-\Phi_{4}^{\exp }\left(t_{j}\right)\right)^{2}
\end{aligned}
$$

where $\Phi_{k}^{\exp }\left(t_{j}\right)=\varphi_{k}\left(t_{j}\right)-\varphi_{3}\left(t_{j}\right)$ with $k=1,2,4$ are the experimental heat fluxes measured by the thermal sensor and $\eta_{j}=\left(Y_{j}, e_{j}, h_{f}, T_{f}, K_{f}\right)$ is the vector of the flame parameters, whose length is equal to 21 if we chose $N=9$. $\alpha_{j}=\Phi_{2}^{\exp }\left(t_{j}\right) / \Phi_{1}^{\exp }\left(t_{j}\right)$ and $\beta_{j}=\Phi_{4}^{\exp }\left(t_{j}\right) / \Phi_{1}^{\exp }\left(t_{j}\right)$ are the weighting coefficients. We used a direct search method to solve the optimisation problem given by equation (4). This method does not require any information about the gradient of the objective function. We employed a special direct search algorithm called the pattern search algorithm, cf. [10,11], that searches a set of points around the current point, looking for one where the value of the objective function is lower than the value at the current point.

\section{Identification by image processing method}

The technique of image processing applied in this study was inspired of that used by Pastor et al. [4]. These authors have used the method of thermal image processing for computing the rate of spread of flame. The images obtained with the thermographic camera allowed to ascertain the temperature corresponding to each pixel in the image. In our case, the visual video camera (30 images/second, $640 \times 480$ pixels) of the fire-fighters was used, and the images obtained were analysed to determine the fire front positions and the rate of spread.

The image processing method developed in this study involved four steps. The first one was to select part of the decompressed video composed of 9 images in propagation zone. The second was to develop a function to compute a matrix of correspondence between the pixels in the image and their positions in the real plane. The image processing poses the problem of the geometrical distortion. Indeed, images cannot possible be recorded perfectly perpendicular to the front of flame. The correspondence between pixels and real points is modified by the angle of distortion. To overcome this problem, a homography matrix $\mathbf{H}$ was determined which defined the correspondence of each image pixels to real positions in the plane. For the plane surfaces, only the correspondence of 4 points A, B, C, and D can be used to define the matrix $\mathbf{H}$ (figure.2).

The matrix $\mathbf{H}$ is calculated by applying the simple direct linear transformation (DLT) algorithm as described by Hartely and Zisserman [7] and used in [4]; it can be written: 


$$
\mathbf{H}=\left[\begin{array}{ccc}
-0.0010 & 0.0005 & -0.0630 \\
0.0000 & 0.0039 & -1.5997 \\
0.0000 & 0.0011 & -0.7684
\end{array}\right]
$$

The third step consists to the research of the best channels of image processing. Each colour image is composed of three black and white images corresponding each one to a coding of a colour (RGB) (figure.3). Then, the test can be done on three different channels, with the index values of the pixel. According to luminosity, the index values of pixel of the black and white image vary between 0 and 255. For the values lower than the defined threshold, the pixels were putted in black (it's meaning that these pixels are not enough luminous to belong to front of flame) and the opposite are putted in white (belong to front of flame). The different channels are tested one after the other in order to define the optimal thresholds for each one. The optimal index value of each channel correspond to the value which allows to eliminate as well as possible the points not belonging to the front of flame (figure.3).

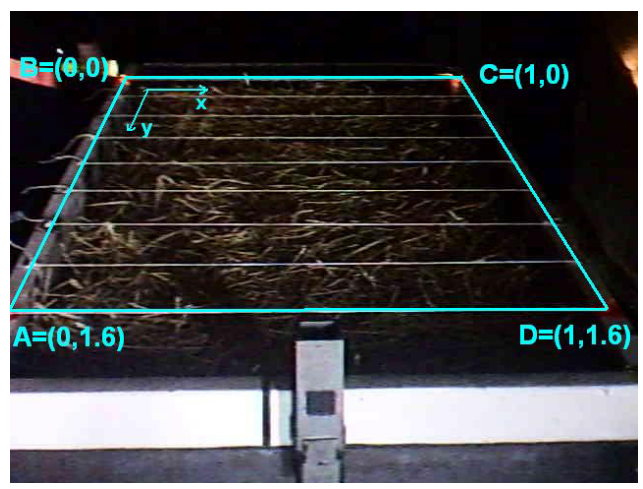

Figure 2: $\quad$ Scheme and dimensions of the study area (propagation zone).

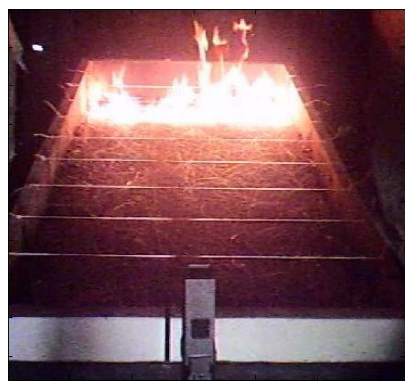

[Original colour image]

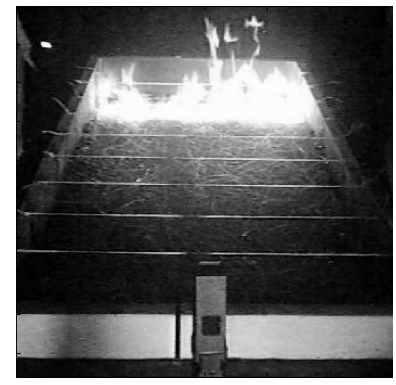

[black and white image from one of channels]

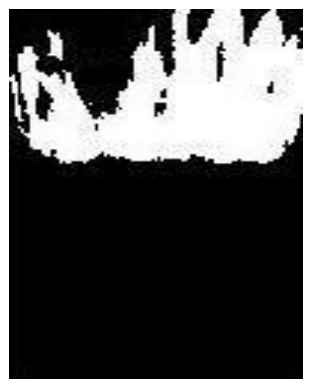

[result of image processing using a threshold of 226]

Figure 3: $\quad$ Example of image processing. 
Once these optimal thresholds are obtained, others tests were carried out with different channels simultaneous. After these tests, we choose the best combination which gives images without points not belonging of the flame front and a good shape of flame front. The pixels in the 9 images are transformed into real coordinates using the matrix $\mathbf{H}$.

The last step is to calculate the positions of fire front. The principle of this step is to realise test on all pixels (column after column) starting with the top of the image, in order to find the position of the last white point in each column, and consequently to store the position of the flame front. Finally, for all images, the associated time and the median $(\mathrm{x}=0.5 \mathrm{~m})$ positions were stored for determining the rate of spread.

\section{Results and discussion}

After the nine images processing we can plot the evolution with time of the different fire front profile in the figure.4. The median positions along the propagation zone corresponding to the middle of the combustion vat with those obtained by the heat fluxes method and their relative uncertainties are presented in table 2 and figure.5. Of course the values of $Y_{\mathrm{j}}$ given by the thermal sensor method have been obtained after the convergence of the minimisation algorithm applied to the objective function described above. Also, the flame characteristics such as the thicknesses $e_{\mathrm{j}}$, the height $h_{\mathrm{f}}$, the temperature $T_{\mathrm{f}}$ and the extinction coefficient $K_{\mathrm{f}}$ are determined by the same method and given in the table 3 .

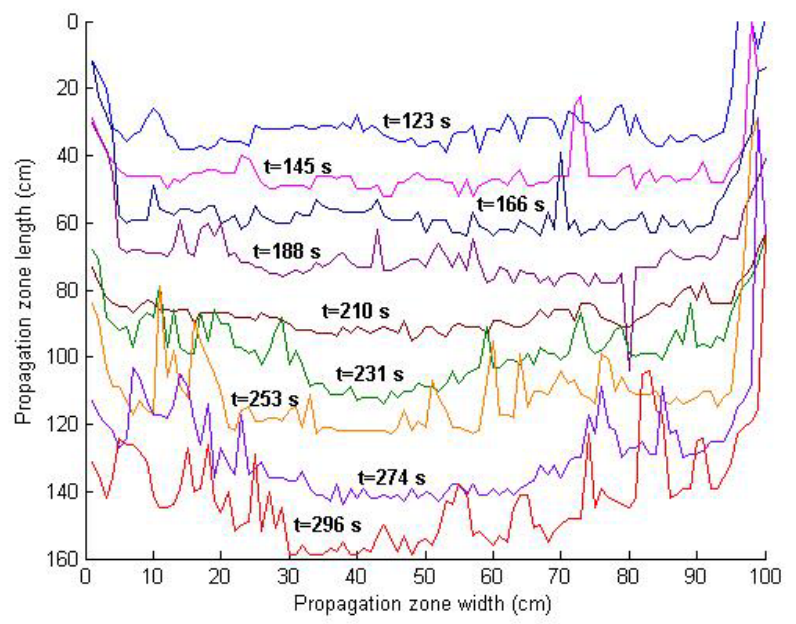

Figure 4: $\quad$ Fire front profile evolution with time.

If we use the parameters of tables 2 and 3 to calculate the theoretical heat fluxes given by equations (2) and (3), we can plot their curves with the experimental heat fluxes in figure.5. We observe a good accord between the theoretical and the experimental heat fluxes for each face of thermal sensor. 
The rates of spread $R O S$ are deduced using a linear regression based on the values of the fire front positions identified by the two methods (Table 2). Figure. 6 shows these linear regressions.

Let us analyse now the different results. The fire front positions values calculated by the thermal sensor method are close to these obtained by image processing. The values of the ratio $\Delta Y_{j} / L_{p}$ and the relative uncertainty of $R O S$ not exceed respectively $7 \%$ and $4 \%$. With these results we can say that the heat fluxes technique is validated by the image processing method.

Table 2: $\quad$ Values of the fire front positions and the rates of spread obtained by the two methods of identification.

\begin{tabular}{|c|c|c|c|c|c|c|c|c|c|}
\hline \multicolumn{10}{|c|}{ Thermal sensor method } \\
\hline$Y_{\mathrm{j}}[\mathrm{cm}]$ & 159.3 & 148.6 & 131.6 & 120.9 & 101.7 & 82.4 & 69.8 & 49.5 & 40.2 \\
\hline $\operatorname{ROS}[\mathrm{cm} / \mathrm{s}]$ & \multicolumn{9}{|c|}{0.72} \\
\hline \multicolumn{10}{|c|}{ Image processing method } \\
\hline$Y_{\mathrm{j}}[\mathrm{cm}]$ & 149.0 & 139.0 & 123.0 & 113.0 & 95.0 & 77.0 & 65.0 & 46.0 & 32.0 \\
\hline $\operatorname{ROS}[\mathrm{cm} / \mathrm{s}]$ & \multicolumn{9}{|c|}{0.69} \\
\hline \multicolumn{10}{|c|}{ Relative uncertainties (\%) } \\
\hline$\Delta Y_{j} / L_{p}$ & 6.4 & 6.0 & 5.4 & 4.9 & 4.2 & 3.4 & 3.0 & 2.2 & 5.1 \\
\hline$\frac{\Delta(R O S)}{R O S}$ & & & & & 4 & & & & \\
\hline
\end{tabular}

Table 3: Values of thicknesses, height, temperature and extinction coefficient of the flame.

\begin{tabular}{|c|c|c|c|c|c|c|c|c|c|c|}
\hline \multicolumn{2}{|c|}{ Flame thicknesses $e_{i}[\mathrm{~cm}]$} & 46 & 45 & 41 & 43 & 42 & 36 & 37 & 32 & 46 \\
\hline \multirow{3}{*}{$\begin{array}{l}\text { Flame } \\
\text { height } h_{\mathrm{f}} \\
{[\mathrm{cm}]}\end{array}$} & Experimental value & & & & & 28 & & & & \\
\hline & Estimated value & & & & & 32 & & & & \\
\hline & $\begin{array}{c}\text { Relative uncertainty } \\
{[\%]}\end{array}$ & & & & & 12.5 & & & & \\
\hline \multicolumn{2}{|c|}{ Flame temperature $T_{\mathrm{f}}\left[{ }^{\circ} \mathrm{C}\right]$} & & & & & 927 & & & & \\
\hline $\begin{array}{l}\text { Flame } \\
\text { extinction }\end{array}$ & Estimated value & & & & & 0.2 & & & & \\
\hline $\begin{array}{l}\text { coefficient } \\
K_{\mathrm{f}}\left[\mathrm{m}^{-1}\right]\end{array}$ & Value of [12] & & & & & 0.2 & & & & \\
\hline
\end{tabular}

The estimated values of flame thicknesses are coherent to the observed values. The relative gap between the experimental and the identified flame height is about $12 \%$. This value is related to the notion of the average flame height used 


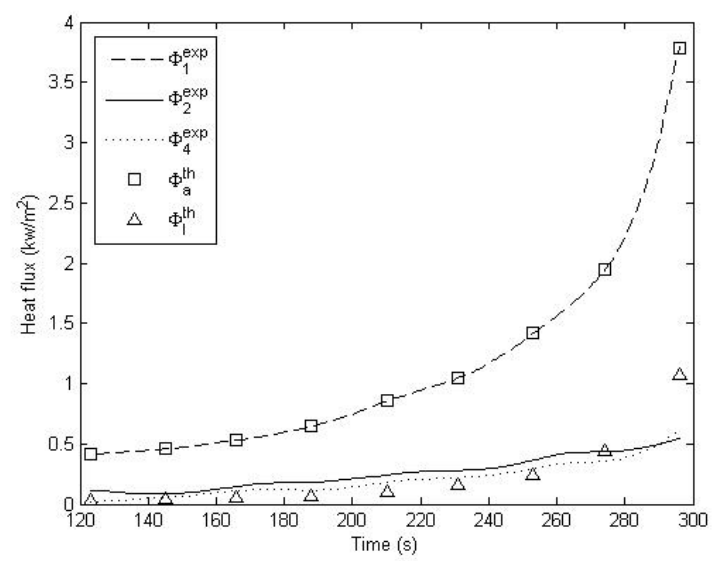

Figure 5: Theoretical and experimental heat fluxes versus time.

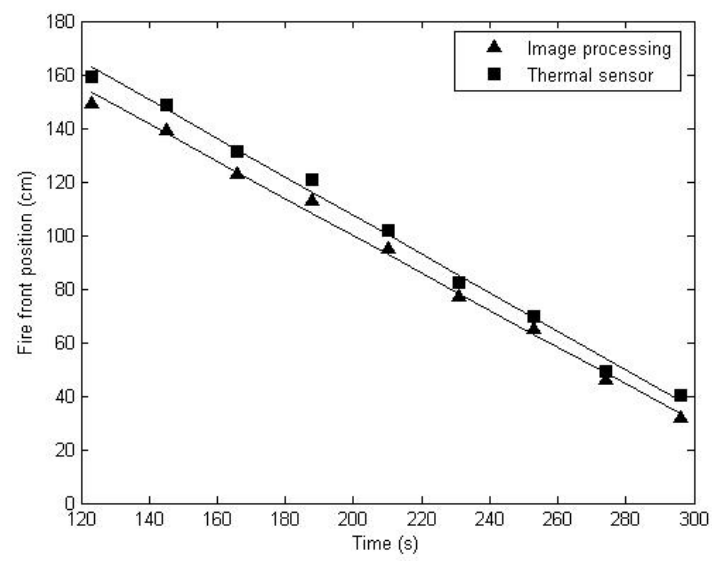

Figure 6: Fire front positions versus time for the two methods.

in forest fire. Indeed, in the experiments of fire the flame height is not constant in the fire front. The extinction coefficient obtained by inverse method is equal to the one given by Margerit and Séro-Guillaume [12].

\section{Conclusion}

We have presented in this paper a new physical method for determining the fire front positions and the flame characteristics using a heat fluxes measurements. Concerning the fire front positions and the rate of spread, this technique gave comparable results with those obtained by the image processing method adapted from the one used by Pastor et al. [4] and presented here. Let us note that this 
technique made it possible to validate the first one. In addition, the application of the inverse method on the thermal measurements of the sensor makes possible also to determine the characteristics of flame such as thicknesses, height, temperature and extinction coefficient. The values of the different parameters obtained in this study are coherent and can be compared favourably to experiment and literature results.

\section{Acknowledgements}

This research has been done under the support of a national project ANR - P.I.F. (Protection contre les Incendies de Forêts). The authors would especially like to acknowledge Philippe BRETON, from the SDIS37, who made valuable recommendations on fire experiments and Emmanuel MENNESSON for his help in designing the experimental set-up.

\section{References}

[1] Balbi, J.H., Rossi, J.L., Marcelli, T. \& Santoni, P.A., A 3D physical realtime model of surface fires across fuel beds. Combustion Science and Technology, 179(12), pp. 2511-2537, 2007.

[2] Séro-Guillaume, O., Ramezani, S., Margerit, J. \& Calogine, D., On large scale forest fires propagation models. International Journal of Thermal Sciences, 47(6), pp. 680-694, 2008.

[3] Porterie, B., Zekri, N., Clerc, J.P. \& Loraud, J.C., Modelling forest fire spread and spotting process with small world networks. Combustion and Flame, 149, pp. 63-78, 2007.

[4] Pastor, E., Àgueda, A., Andrade-Cetto, J., Muñoz, M., Pérez, Y. \& Planas, E., Computing the rate of spread of linear flame fronts by thermal image processing. Fire Safety Journal, 41, pp. 569-579, 2006.

[5] Dupuy, J.L., Vachet, P. \& Maréchal, J., Fuel bed temperature measurements in laboratory fires spreading over a slope. $V$ International Conference on Forest Fire Research, D.X. Viegas (Ed), 2006.

[6] Mendes-Lopes, J.M.C. \& Ventura, J.M.P., Flame characteristics in fires propagating in beds of pinus halepensis needles. $V$ International Conference on Forest Fires Research, D.X. Viegas (Ed), 2006.

[7] Hartley, R. \& Zisserman, A., Multiple view geometry in computer vision, 2nd ed. Cambridge: Cambridge University Press, 2003.

[8] Chetehouna, K., Séro-Guillaume, O., Sochet, I., \& Degiovanni, A., On the experimental determination of flame front positions and of propagation parameters for a fire. International Journal of Thermal Sciences, doi:10.1016/j.ijthermalsci.2007.10.006, 2007.

[9] Chetehouna, K., Séro-Guillaume, O., Degiovanni, A., Picard, C. \& Giroud, F., Study of a thermal sensor intended for the calibration of propagation models of forest fires. Proceedings of Inverse problems and experimental design in thermal and mechanical engineering (Eurotherm Seminar 68), Edizioni Ets, Poitiers, France, pp. 287-292, 2001. 
12 Modelling, Monitoring and Management of Forest Fires I

[10] Michael, L.R. \& Torczon, V., Pattern Search Algorithms for Bound Constrained Minimization. SIAM Journal on Optimization, 9(4), pp. 10821099, 1999.

[11] Audet, C. \& Dennis Jr., J.E., Analysis of Generalized Pattern Searches. SIAM Journal on Optimization, 13(3), pp. 889-903, 2003.

[12] Margerit, J. \& Séro-Guillaume, O., Modelling forest fires. Part II: reduction to two-dimensional models and simulation of propagation. International Journal of Heat and Mass Transfer, 45(8), pp. 1723-1737, 2002. 